\title{
Narrow Bandwidth Single-Resonator MEMS Tuning Fork Filter
}

\author{
Jize Yan, Ashwin A. Seshia \\ Department of Engineering \\ University of Cambridge, \\ Cambridge, UK
}

\begin{abstract}
We present a solution for a fourth-order, narrowbandwidth filter comprising of a single silicon tuning fork resonator driven using one electrode only. Voltage controlled electrical spring tuning is employed to match the primary and secondary modes of the resonator to achieve filter response. A narrow bandwidth single resonator MEMS tuning fork filter is demonstrated with a center frequency of $1.2866 \mathrm{MHz}$, a $3 \mathrm{~dB}$ bandwidth of $0.0085 \%$ and a $1.5 \mathrm{~dB}$ ripple.
\end{abstract}

\section{INTRODUCTION}

Electromechanical filters based on microfabricated silicon resonators have the potential to replace quartz crystal and SAW technologies with significant benefits in terms of reduced size, power dissipation and the potential for integration with conventional CMOS technologies [LHP98] $[\mathrm{GKL}+06][\mathrm{LLX}+05]$. Reported results to achieve higher-order and narrow-bandwidth silicon microfabricated filters are usually based on the mechanical and/or electrical coupling of MEMS resonator arrays [LHP98]. For instance, micro-electromechanical resonators may be coupled via mechanical beams or via electrodes for band-pass filtering functionality [LHP98, PA05]. However, the design and fabrication of narrow bandwidth and high frequency filters remains a challenge, particularly with increasing center frequency due to the demands on the miniaturization of both the resonator as well as the coupling elements. One possible solution is to electrically couple serially-arranged arrays of resonators [PA05] to overcome the demands on fabrication tolerances for coupling elements in high frequency microresonator filters. A second solution [DN05] achieves fourth-order filter response by adding frequency control masses to break the degeneracy of two closely spaced resonant modes in a single structure. However, this solution suffers the drawback of large electrode count and the requirement to precisely preset the frequency control masses to tune the filter bandwidth.

We propose a solution for a fourth-order, narrowbandwidth filter comprising of a single silicon tuning fork resonator driven using one electrode only. By varying the DC voltage on the electrode, it is possible to employ electrostatic spring tuning to lower the frequency of the out-of-phase tuning fork mode to closely match the in-phase mode. As the frequencies of the two modes are gradually matched, there is

\author{
Kim L. Phan, Peter G. Steeneken, Joost T.M. van Beek \\ NXP Semiconductors, \\ Philips Campus, \\ Eindhoven, The Netherlands
}

an increased coupling of mechanical energy between the two modes through the stem of the tuning fork ultimately resulting in a narrow bandwidth filter response.

\section{CAPACITIVE TRANSDUCTION AND FILTER MECHANISM}

\section{A. Tuning forks}

Tuning forks are utilized as a standard frequency benchmark for the tone-calibration of musical instruments at the macro-scale and have been demonstrated as micro-scale building blocks in oscillators [Roe98], accelerometers [RHPS95] and gyroscopes [PGR96]. An SEM of the tuning fork filter is shown in Fig. 1. The single-ended tuning fork consists of two tines attached through a single stem with the nodal point of vibration set at the support stem [Joh83].

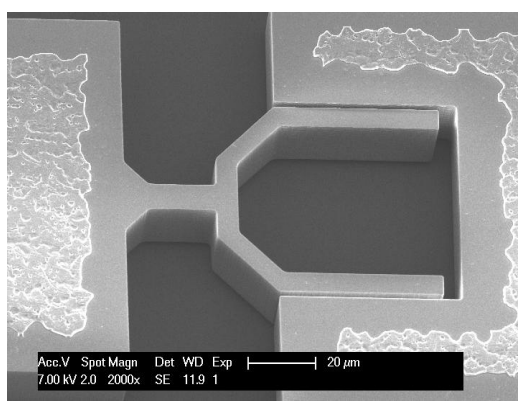

Fig. 1. SEM of the single-ended tuning fork (SETF) filter

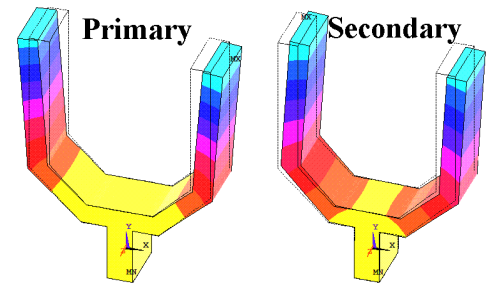

Fig.2. FEA is used to depict the primary mode (symmetric coupled mode) and the secondary mode (asymmetric decoupled mode). 


\section{B. Capacitive actuation}

The tuning fork actuation force $F$ is calculated from the derivative of the potential energy with respect to the resonator displacement $x$, as [Sen01][Ngu94]

$$
\begin{aligned}
& F=\frac{d P . E .}{d x}=\frac{1}{2} V^{2} \frac{\partial C}{\partial x}=\frac{1}{2}\left(V_{D C}+V_{A C} \cos \omega t\right)^{2} \frac{\partial C}{\partial x} \\
& =\left(\frac{V_{D C}^{2}}{2}+\frac{V_{A C}^{2}}{4}+V_{D C} V_{A C} \cos \omega t+\frac{V_{A C}^{2}}{4} \cos 2 \omega t\right) \frac{\partial C}{\partial x}(1)
\end{aligned}
$$

If the applied DC voltage $\left(V_{D C}\right)$ is much larger than the $\mathrm{AC}$ voltage $\left(V_{A C}\right)$, the 2 nd harmonic term in the above equation is small in comparison with fundamental. Under these conditions, the 1st harmonic term will dominate the driving force at the resonant frequency and can be written as:

$$
F=V_{D C} V_{A C} \frac{\partial C}{\partial x} \cos \omega t
$$

\section{Narrow bandwidth Filter mechanism}

As opposed to adding frequency control masses to break the degeneracy of orthogonal modes of a micromechanical disk as reported in [DN05], voltage control is used to shift the frequency of the primary mode (symmetric coupled mode) preferentially towards the secondary mode (asymmetric decoupled mode) of the tuning fork filter as shown in Fig 3. The electrical energy is pumped preferentially into the primary mode of the resonator while the stem couples mechanical energy between the two modes. The electrical effective spring constant $\left(k_{\text {ele }}\right)$ is given by [Sen01]:

$$
\frac{1}{2} k_{\text {ele }} g^{2}=\frac{1}{2} C_{0} V_{D C}^{2} \rightarrow k_{\text {ele }}=\frac{C_{0} V_{D C}^{2}}{g^{2}}
$$

where $C_{0}$ is the nominal parallel-plate capacitance and $g$ is the nominal gap between the electrode and the resonator. The overall effective spring constant $\left(k_{\text {eff }}\right)$ is given by [LLL+04]

$$
k_{\text {eff }}=k_{\text {mech }}-k_{\text {ele }}
$$

The resonant frequency $(f)$ as a function of DC bias voltage is given by

$$
f=\frac{1}{2 \pi} \sqrt{\frac{k_{e f f}}{m_{e q}}} \approx f_{0}-\frac{C_{0} V_{D C}^{2}}{4 \pi m_{e q} g^{2}}
$$
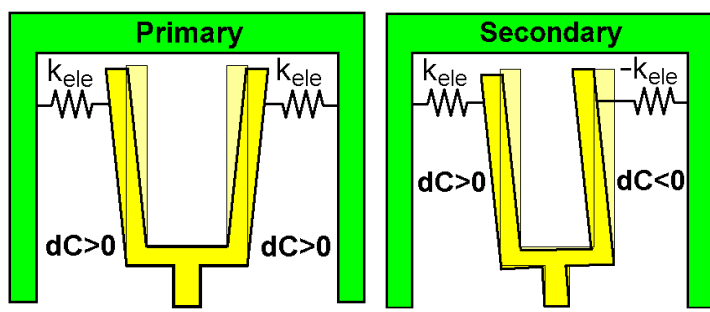

Fig.3. The electrode topology allows for a direct capacitive actuator interface to the primary mechanical mode.
The driving force expressions for the primary $\left(F_{p r i}\right)$ and secondary modes $\left(F_{s e}\right)$ using the electrode topology shown in Fig. 3 are given by:

$$
F_{p r i}=V_{D C} V_{A C} \frac{C_{0}}{g} \cos \omega t>F_{s e}=V_{D C} V_{A C} \frac{C_{0} x}{g^{2}} \cos \omega t \text { (6) }
$$

As can be seen from Eq. 6, electrical energy is pumped preferentially into the primary mode. Similarly, the electrical spring softening preferentially modulates the frequency of the primary mode. The frequency softening effect of the primary and secondary modes are given as

$$
\begin{aligned}
& f_{p r}^{2}=f_{p r 0}^{2}-\frac{\mathcal{E}_{0} A_{e l e} V_{D C}^{2}}{4 \pi^{2}(g-x)^{3} m_{p r i}} \\
& f_{\mathrm{se}}^{2}=f_{\mathrm{se} 0}^{2}-\frac{\varepsilon_{0} A_{e l e} V_{D C}^{2}}{4 \pi^{2} m_{\mathrm{sec}}}\left(\frac{1}{(g-x)^{3}}-\frac{1}{(g+x)^{3}}\right)
\end{aligned}
$$

The linear relationship between primary mode frequency shift and the square of the DC voltage bias as predicted by the analytical model is confirmed by experiment as shown in Fig. 4. A comparison between the experimental results and the calculated electrical-spring-softening effect for the primary and secondary modes is shown in Fig. 5.

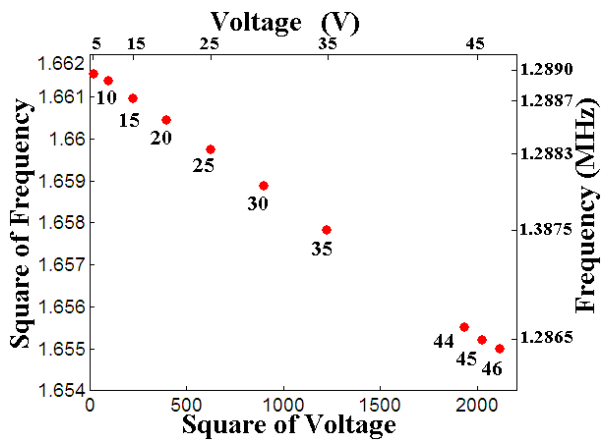

Fig. 4. Measured electrical spring softening induced frequency shift of the primary mode of the SETF resonator with the squared of the DC bias voltage.

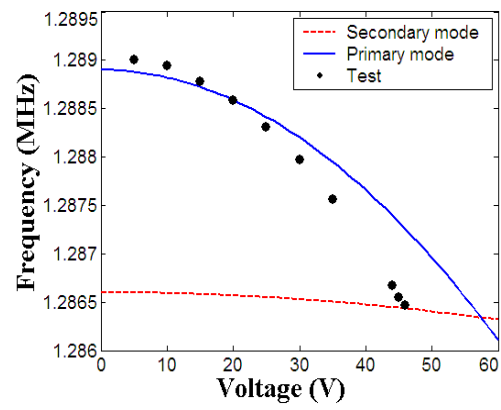

Fig. 5. Comparison of the measured and modeled resonant frequency with varying DC-bias for the primary and secondary modes.

\section{Equivalent circuit model and Y-parameters extraction}

The measured resonator characteristics are typically affected by parasitic capacitances in parallel and series with 
the resonator as shown in Fig. 6. In the limit of high $Q$-factor, the parallel resonant peak is close to the series resonant frequency [KMO+03]. The measured S-parameters are fitted to the electrical equivalent circuit model (shown in Fig. 6) to extract the motional parameters of the resonator.

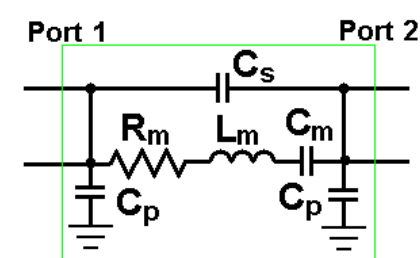

(a) With DC-bias

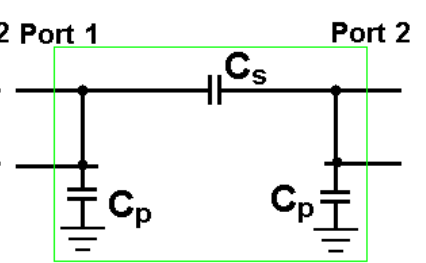

(b) Without DC-bias
Fig. 6. Two port network model of the SETF

The equivalent resonator motional parameters are given by:

$$
R_{m}=\frac{\sqrt{k m}}{Q \eta^{2}}, C_{m}=\frac{\eta^{2}}{k}, L_{m}=\frac{m}{\eta^{2}}, \eta=V_{D C} \frac{\partial C}{\partial x}
$$

Where $R_{m}, C_{m}$ and $L_{m}$ are the motional resistance, capacitance and inductance respectively, $\eta$ is the transduction parameter and $Q$ is the Quality factor of resonance. The equivalent circuit model can be represented as Fig. 6.b when the bias voltage is removed. In this case:

$$
\eta=0, R_{m}=\infty, C_{m}=0, L_{m}=\infty
$$

The measured $Y_{21}$ parameter with and without applied DC bias can be related to the motional parameters of the resonator and the capacitive parasitics as:

$$
\begin{aligned}
& Y_{21}\left(V_{D C}\right)=-\frac{1}{R_{m}+1 / j \omega C_{m}+j \omega L_{m}}-j \omega C_{s} \\
& Y_{21}(0)=\left.\frac{I_{2}}{V_{1}}\right|_{V_{2}=0}=-j \omega C_{s}
\end{aligned}
$$

Thus the motional impedance of the resonator can be derived from these measurements as:

$$
R_{m}+1 / j \omega C_{m}+j \omega L_{m}=\frac{1}{Y_{21}(0)-Y_{21}\left(V_{D C}\right)}
$$

\section{E. Nonlinear effects}

A number of experiments were conducted to estimate limiting nonlinearities for various driving voltages. Fig. 7 shows the measured frequency response of the resonator with varying DC voltage at fixed AC input power. Fig. 8 shows the extracted Y-parameters with variable DC bias for two different values of $\mathrm{AC}$ driving $-5 \mathrm{dBm}$ and $5 \mathrm{dBm}$ respectively. The Y-parameters are also extracted for various AC drive voltages as shown in Fig. 9. A classic spring softening Duffing nonlinearity limits the output amplitude for increasing $\mathrm{AC}$ input power and DC bias [LL60].

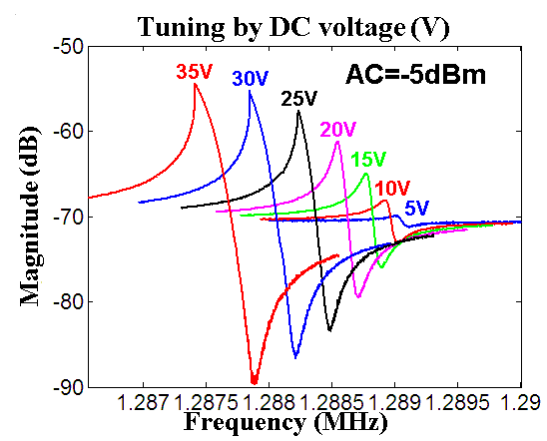

Fig. 7. SETF resonator S-parameters measurement results with fixed $\mathrm{AC}$ power at $-5 \mathrm{dBm}$ and variable $\mathrm{DC}$ bias.
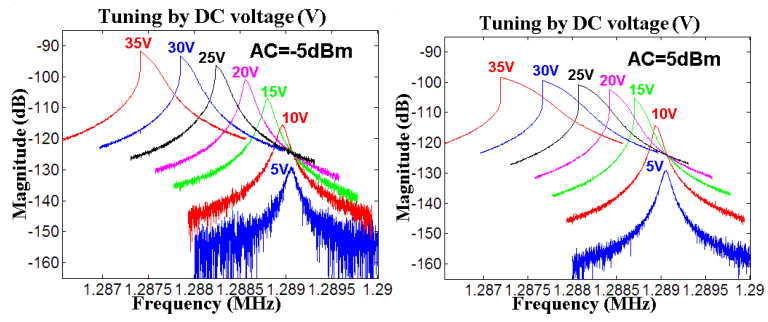

Fig. 8. Extracted Y-parameters frequency response with variable $\mathrm{DC}$ bias and fixed $\mathrm{AC}$ power at $-5 \mathrm{dBm}$ and $5 \mathrm{dBm}$ respectively.

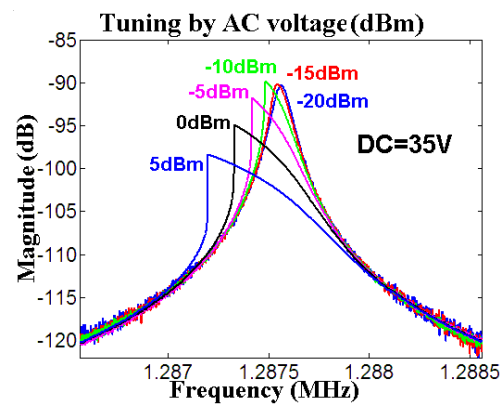

Fig. 9. Extracted Y-parameters frequency response with variable $\mathrm{AC}$ power and for resonator DC-bias fixed at $35 \mathrm{~V}$.

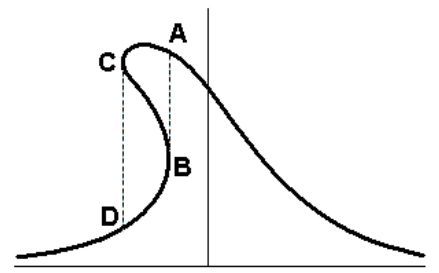

Fig. 10. Typical frequency response for a resonator describing a spring softening Duffing nonlinearity. 
Fig. 10 depicts the frequency response of a nonlinear resonator depicting a Duffing spring softening type of nonlinearity. [KMOS03]. A clear hysteresis is observed together in the frequency response together with an amplitude jump as the frequency is scanned forwards and backwards about resonance. The critical driving force FCR is determined when the section $\mathrm{BC}$ (in Fig. 10) is reduced to a point which is given by [LL60]:

$$
F_{C R}=\sqrt{8 m^{2} \omega_{0}^{2} \lambda^{3} /|\kappa|}
$$

where $m$ is the effective mass, $\omega_{0}$ is the resonant frequency without nonlinear effect and $\lambda$ is the damping factor. Substituting an expression for driving force as a function of driving voltage from Eq. (2), the unstable nonlinear vibration boundary is determined as:

$$
V_{D C} V_{A C} \frac{\partial C}{\partial x}=\sqrt{8 m^{2} \omega_{0}^{2} \lambda^{3} /|\kappa|}
$$

Thus, a condition is derived that defines a ceiling on the product of the applied DC and AC voltage for a given resonator topology to operate in the linear regime of the resonator $[\mathrm{ACC}+06]$.

\section{FILTER PERFORMANCE}

The measured frequency response of the SETF is shown in Fig. 11 as a function of varying DC bias voltage. As the frequency of the two modes of the SETF are tuned closer, a filter characteristic is obtained at a bias voltage close to $45 \mathrm{~V}$.
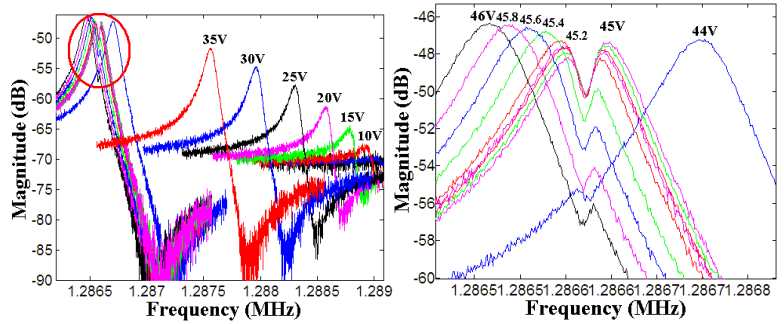

Fig.11 Measurement results of variable DC-voltage tuning.

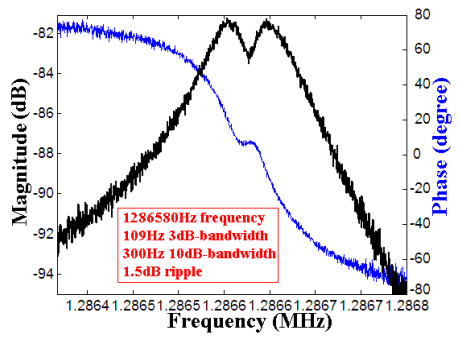

Fig. 12. Magnitude and Phase S-parameter measurements for the SETF filter. The input AC power is $-20 \mathrm{dBm}$ and DC bias is $45 \mathrm{~V}$.

Fig. 12 shows the measured S-parameters of the filter, where the central frequency is $1.2866 \mathrm{MHz}$, the $3 \mathrm{~dB}$-bandwidth is $0.0085 \%(109 \mathrm{~Hz}) 10 \mathrm{~dB}-$ bandwidth is $0.023 \%(300 \mathrm{~Hz})$ and the ripples are $1.5 \mathrm{~dB}$ with $45.08 \mathrm{~V}$ DC tuning voltage. The high insertion loss is due to the large impedance mismatch between the resonator and the measurement setup. Dimensional scaling (including decreasing the capacitive gaps) will allow for higher frequency SETF filters to be realized using this concept while simultaneously lowering the insertion loss as well.

\section{REFERENCES}

[1] [LHP98] L. Lin, R.T. Howe, A.P. Pisano, 'Microelectromechanical Filters for Signal Processing,' J. Microelectromech. Syst., vol. 7, pp. 286-294, 1998.

[2] [GKL+06] D.Galayko, A.Kaiser, B.Legrand, L.Buchaillot, C.Combi, D.Collard, 'Coupled-resonator micromechanical filters with voltage tuneable bandpass characteristic in thick-film polysilicon technology,' Sensors and Actuators A. vol.126, pp. 227-240, 2006

[3] [LLX+05] S.-S. Li, Y.-W. Lin, Y. Xie, Z. Ren, C. T.-C. Nguyen, 'Small percent bandwidth design of a 431-MHz notch-coupled micromechanical hollow-disk ring mixer-filter,' Proceedings, IEEE Int. Ultrasonics Symposium, Sept. 18-21, pp.1295-1298, 2005.

[4] [PA05I] S. Pourkamali, F. Ayazi, 'Electrically coupled MEMS bandpass filters Part I: With coupling element,' Sensors and Actuators A. vol.122, pp.307-316, 2005

[5] [PA05II] S. Pourkamali, F. Ayazi, 'Electrically coupled MEMS bandpass filters Part II. Without coupling element,' Sensors and Actuators A. vol.122, pp.317-325, 2005

[6] [DN05] M. U. Demirci and C. T.-C. Nguyen, 'Single-resonator fourthorder micromechanical disk filters,' Proceedings, 18th Int. IEEE Micro Electro Mechanical Systems Conf., Jan. 30 - Feb. 3, pp. 207-210, 2005.

[7] [NNM79] Mitsuo Nakazawa, Yatsuka Nakamura, Shigemitsu Miyashita, 'Frequency-Temperature Characteristics of Quartz Crystal Flexure Bars and Quartz Crystal Tuning Forks,' IEEE Transactions on Sonics and Ultrasonics, Vol. SU-26, No. 5, 1979, pp. 369-376

[8] [Roe98] T.A. Roessig, 'Integrated MEMS Tuning Fork Oscillators for Sensor Applications', PhD thesis, University of Califonia, Berkeley, 1998

[9] [RHPS95] T.A. Roessig, R.T. Howe, A.P. Pisano, J.H. Smith, 'Surfacemicromachined resonant force sensor', Proceedings of ASME Dynamic Systems and Control Division, pp. 871-876, 1995

[10] [PGR96] F. Paoletti, M.-A. Gretillat, N.F. de Rooij ,'A silicon micromachined tuning fork gyroscope' IEE Colloquium on Silicon Fabricated Inertial Instruments, London, UK, pp.3/1-3/6, 2 Dec 1996

[11] [Joh83] R.A. Johnson, 'Mechanical filters in electronics,' John Wiley \& Sons, 1983

[12] [Sen01] Stephen D. Senturia, Microsystem Design, Kluwer Academic Publishers, 2001

[13] [Ngu94] C.T.-C. Nguyen, 'Micromechanical Signal Processors,' PhD thesis, Univ. of California, Berkeley, 1994

[14] [LLL+04] Yu-Wei Lin, Seungbae Lee, Sheng-Shian Li, Yuan Xie, Zeying Ren, Clark T.-C. Nguyen, 'Series-Resonant VHF Micromechanical Resonator Reference Oscillators,' IEEE Journal of Solid-State Circuits, vol. 39, no. 12, pp. 2477-2491, 2004

[15] [HHP98] D. A. Horsley, R. Horowitz and A. P. Pisano, 'Microfabricated Electrostatic Actuators for Hard Disk Drives,' IEEE/ASME Transactions on Mechatronics, Vol. 3, No. 3, pp. 175-183, Sept. 1998.

[16] [Kel00] S. Graham Kelly, 'Fundamentals of Mechanical Vibrations, 2nd edition' McGraw-Hill Higher Education, 2000

[17] [KMOS03] Kaajakari, V, Mattila, T, Oja, A, Seppa, H, 'Nonlinear Limits for Single Crystal Silicon Microresonators', J. Microelectromechanical Systems, vol. 12, no. 12, no. 5, pp. 714-725. 2003

[18] [LL60] L.D. Landau and E.M. Lifshitz, 'Mechanics,' Oxford, 1960

[19] [ACC+06] M. Agarwal, S. A. Chandorkar, R. N. Candler, B. Kim, M. A. Hopcroft, R. Melamud, C. M. Jha, T. W. Kenny, B. Murmann, 'Optimal drive condition for nonlinearity reduction in electrostatic microresonators,' Applied Physics Letters 89, 214105, 2006. 